\title{
Highly Efficient Synthesis of New 2H-chromene Dyes Using Cu-SBA-15
}

\section{SHIRIN FARAJI KAMAZANI*, SIAVASH SALEK SOLTANI and AFSANEH ZONOUZI}

School of Chemistry, University College of Science, University of Tehran, Tehran, Iran.

${ }^{*}$ Corresponding author E-mail: Shirinfaraji58@gmail.com

http://dx.doi.org/10.13005/ojc/320525

(Received: August 01, 2016; Accepted: September 30, 2016)

\begin{abstract}
In this work, some new azotated $2 \mathrm{H}$-chromene derivatives were successfully synthesized by use of mesoporous Cu-SBA-15 as nanocatalyst leading short reaction times and high yields. By this research, the scope of azo compounds was increased.
\end{abstract}

Keywords: Azo dye, Chromene, Mesoporous, Catalyst. Cu-SBA-15.

\section{INTRODUCTION}

$2 \mathrm{H}$-chromenes are very important heterocyclic compounds which have shown biological activities ${ }^{1-4}$, some medical properties such as antihypertensive ${ }^{5}$ and cardio protectors as well as hypoglycemic agents $s^{6,7}$. Some of them are insecticide compounds ${ }^{8}$. In the recent years, they have been used in sensors for detection of metals and some compounds with high selectivity ${ }^{9,10}$. Until now, diverse methods have been published for the synthesis of $2 \mathrm{H}$-benzopyran derivatives ${ }^{11-17}$. They are also good intermediates that have been applied in several organic synthesis ${ }^{18-20}$.

Azo compounds are very considerable in dyes and pigments industry ${ }^{21}$. They are the main class of all synthetic dyes that are widely used in the world ${ }^{22}$. The importance of azo dyes returns to their significant physicochemical features such as stability $^{23}$, optical propertie ${ }^{24,25}$ and their extensive applications in chemosensors ${ }^{26,27}$, liquid crystals $^{28}$ and nanotubes ${ }^{29}$.

Recently, an enhancing attention has been concentrated on the development of new synthetic paths in processes employing functionalized mesoporous catalysts ${ }^{30}$.

Mesoporous material has been grown in academia because of its extraordinary surface area, narrow size distribution of porosity, heat resistance and mechanical stability that make them outstanding catalysts ${ }^{31,32}$. 
In this work, we use them for the synthesis of new class of azo dyes in very good conditions.

\section{EXPERIMENTAL}

\section{General information}

In this research, 5-Phenylazo-salicylaldehyde 1 was synthesized similar to the reported methods ${ }^{33,34}$, but optimized, and also the mesoporous Cu-SBA-15 catalyst was prepared by literature ${ }^{35}$.

All of the chemicals and solvents were purchased from Merck (Germany), Sigma-Aldrich (United States) and Fluka (Switzerland) corporations. They were used without anymore purification. TLC was performed on precoated plastic sheets $\left(25 \mathrm{DC} \mathrm{UV}-254_{1}\right)$. Melting points totally were weighed on an electrothermal Gallenkamp melting point apparatus and are not corrected. Elemental analysis for $\mathrm{C}, \mathrm{H}$ and $\mathrm{N}$ were done using a Thermo Finnigan Flash EA1112 instrument. IR spectra were obtained on a Shimadzu FT-IR-4300 spectrophotometer as $\mathrm{KBr}$ discs. ${ }^{1} \mathrm{HNMR}$ and ${ }^{13} \mathrm{CNMR}$ spectra were analyzed on a Bruker $300 \mathrm{MHz}$ spectrometer (but 2a, $2 \mathrm{c}$ and $2 \mathrm{~d}$ compounds were analyzed on a Bruker $250 \mathrm{MHz}$ spectrometer) in $\mathrm{CDCl}_{3}$, and DMSO- $\mathrm{d}_{6}$, solutions and chemical shifts were noted in $\delta$ units by using TMS, as standard.

\section{General procedure for the synthesis of 5-Phenylazo-salicylaldehyde (1)}

To a solution of substituted aniline (0.05 $\mathrm{mol}$ ) in a small portion of water was slowly added $7 \mathrm{ml}$ of $37 \%$ aq HCl solution at $0-5^{\circ} \mathrm{C}$ with rapid stirring. $15 \mathrm{ml}$ of $5 \mathrm{M}$ aq NaNO$_{2}$, solution was added dropwise to afford yellowish diazonium salts. Then the solution of salicylaldehyde (3-methoxy salicylaldehyde, 0.05 $\mathrm{mol}$ ) in $2 \%$ aq $\mathrm{KOH}$ solution $(100 \mathrm{~mL}$ ) was added very slowly in 1 hour. The mixture was stirred for 30 minutes. The brown solid was filtered and washed with sufficient water. Finally it was recrystallized from ethanol to get the pure product.

Synthesis of 1-(2-Hydroxy-2-methyl-6 (phenyldiazenyl)-2H-chromene-3-yl) ethanone (2a)

In the mixture of 5-phenylazo-salicylaldehide 1a $(1.13 \mathrm{~g}, 5 \mathrm{mmol})$ and acetylacetone $(0.52 \mathrm{~g}$, $5 \mathrm{mmol})$ in $25 \mathrm{ml}$ solvent $\left(\mathrm{CH}_{2} \mathrm{Cl}_{2} / \mathrm{EtOH} ; 2: 1\right)$, the catalyst Cu-SBA-15 (5 mol\%) was added under rapid stirring in ice bath. The reaction mixture was stirred for 1 hour in $0^{\circ} \mathrm{C}$. The solution was remained in refrigerator for 12 hours. Then, the solvent was evaporated with vacuum rotary. Afterward, the precipitate was washed with sufficient n-hexane.

In order to recovery of the catalyst, the product was dissolved in acetonitrile $(10 \mathrm{ml})$ and shaken for $5 \mathrm{~min}$. then the catalyst was filtered and washed with enough ethyl acetate and the filtrate was evaporated to gain crude product. It was recrystallized from ethanol to obtain orange pure crystals, $\mathrm{mp}, 134-136{ }^{\circ} \mathrm{C}$.

The similar procedure was used leading $2 b-f$.

\section{Representative spectral data}

1-(2-Hydroxy-2-methyl-6-(phenyldiazenyl)-2Hchromene-3-yl) ethanone (2a)

IR $(\mathrm{KBr})\left(v_{\max }, \mathrm{cm}^{\prime \prime 1}\right): 3473,1653,1431$, 1163, 1083. ${ }^{1} \mathrm{HNMR}\left(250 \mathrm{MHz}\right.$, DMSO): $\delta_{\mathrm{H}} 1.87(3 \mathrm{H}$, $\left.\mathrm{s}, \mathrm{CH}_{3}\right), 2.47\left(3 \mathrm{H}, \mathrm{s}, \mathrm{OCH}_{3}\right), 4.4(1 \mathrm{H}, \mathrm{brs}, \mathrm{OH}), 7.07$ $\left(1 \mathrm{H}, \mathrm{d}, \mathrm{J}=8.75 \mathrm{~Hz}, \mathrm{H}_{8}\right), 7.46-7.87(7 \mathrm{H}$, Ar Protons), $7.97\left(1 \mathrm{H}, \mathrm{dd}, \mathrm{J}=8.5 \mathrm{~Hz}, \mathrm{~J}=2.5 \mathrm{~Hz}, \mathrm{H}_{7}\right) \cdot{ }^{13} \mathrm{CNMR}$ (62 MHz, DMSO): $\delta_{\mathrm{C}} 32.5$ (CH3), 32.6 (CH3), 111.3, $122.5,125.1,127.6,128.6,131.9,134.7,136.3$, 139.2, 140, 151.5, 157.2, 161.2 (Alkene and Aryl carbons), $201.6(\mathrm{C}=\mathrm{O})$.

1 - ( 2 - H y d r ox y - 8 - m e t h ox y - 2 - m e t h y l - 6 (phenyldiazenyl)-2H-chromene-3-yl) ethanone (2b)

IR $(\mathrm{KBr})\left(v_{\max }, \mathrm{cm}^{\prime \prime 1}\right): 3472,1665,1431$, 1122, 1072. ${ }^{1} \mathrm{HNMR}(300 \mathrm{MHz}, \mathrm{DMSO}): \delta_{\mathrm{H}} 1.89(3 \mathrm{H}$, $\left.\mathrm{s}, \mathrm{CH}_{3}\right), 2.42\left(3 \mathrm{H}, \mathrm{s}, \mathrm{OCH}_{3}\right), 3.32(1 \mathrm{H}$, brs, $\mathrm{OH}), 3.89$ $\left(3 \mathrm{H}, \mathrm{s}, \mathrm{OCH}_{3}\right), 7.72\left(1 \mathrm{H}, \mathrm{s}, \mathrm{H}_{4}\right), 7.52-7.89(7 \mathrm{H}, \mathrm{Ar}$ Protons). ${ }^{13} \mathrm{CNMR}$ (75 MHz, DMSO): $\delta_{\mathrm{C}} 33.1(\mathrm{CH} 3)$, $32.9(\mathrm{CH} 3), 59.7\left(\mathrm{OCH}_{3}\right), 111.1,113.7,127.1,127.9$, 132.2, 133.8, 135.5, 139.6, 141.1, 149.8, 154.1, 155.2, 156.1 (Alkene and Aryl carbons), 200.3 $(\mathrm{C}=\mathrm{O})$.

1-(2-Hydroxy-2-methyl-6-(p-tolyldiazenyl)-2Hchromene-3-yl) ethanone (2c)

IR $(\mathrm{KBr})\left(v_{\max }, \mathrm{cm}^{\prime \prime 1}\right): 3383,1664,1422$, 1142, 1077. ${ }^{1} \mathrm{HNMR}(250 \mathrm{MHz}, \mathrm{DMSO}): \delta_{\mathrm{H}} 1.85(3 \mathrm{H}$, $\left.\mathrm{s}, \mathrm{CH}_{3}\right), 2.42\left(3 \mathrm{H}, \mathrm{s}, \mathrm{CH}_{3}\right), 2.85\left(3 \mathrm{H}, \mathrm{s}, \mathrm{CH}_{3}\right), 3.1(1 \mathrm{H}$, brs, OH), $7.10\left(1 \mathrm{H}, \mathrm{d}, \mathrm{J}=9 \mathrm{~Hz}, \mathrm{H}_{8}\right), 7.36(2 \mathrm{H}, \mathrm{d}, \mathrm{J}=$ $\left.8 \mathrm{~Hz}, \mathrm{H}_{10}\right), 7.81\left(2 \mathrm{H}, \mathrm{d}, \mathrm{J}=7.75 \mathrm{~Hz}, \mathrm{H}_{9}\right), 7.91-7.93$ $\left(2 \mathrm{H}, \mathrm{m}, \mathrm{H}_{4,7}\right), 8.03\left(1 \mathrm{H}, \mathrm{d}, \mathrm{J}=2.5 \mathrm{~Hz}, \mathrm{H}_{\mathrm{b}}\right) \cdot{ }^{13} \mathrm{CNMR}$ 
(62 MHz, DMSO): $\delta_{\mathrm{C}} 25.7\left(\mathrm{CH}_{3}\right), 32.9(\mathrm{CH} 3), 33.2$ (CH3), 110.8, 123.0, 125.1, 126.9, 128.2, 132.6, 134.0, 135.5, 139.8, 140.3, 149.7, 156.5, 160.7 (Alkene and Aryl carbons), $201.9(\mathrm{C}=\mathrm{O})$.

1-(6-((4-ethylphenyl)diazenyl)-2-hydroxy-2methyl-2H-chromene-3-yl) ethanone (2d)

IR $(\mathrm{KBr})\left(v_{\max }, \mathrm{cm}^{-1}\right): 3291,1654,1411$, 1141, 1078. ${ }^{1} \mathrm{HNMR}(250 \mathrm{MHz}, \mathrm{DMSO}): \delta_{\mathrm{H}} 1.23$ $\left(3 \mathrm{H}, \mathrm{t}, \mathrm{J}=7.5 \mathrm{~Hz}, \mathrm{CH}_{3}\right), 1.87\left(3 \mathrm{H}, \mathrm{s}, \mathrm{CH}_{3}\right), 2.44$ $\left(3 \mathrm{H}, \mathrm{s}, \mathrm{CH}_{3}\right), 2.70\left(2 \mathrm{H}, \mathrm{q}, \mathrm{J}=7.5 \mathrm{~Hz}, \mathrm{CH}_{2}\right), 3.1(1 \mathrm{H}$, brs, OH), $7.10\left(1 \mathrm{H}, \mathrm{d}, \mathrm{J}=8.75 \mathrm{~Hz}, \mathrm{H}_{8}\right), 7.42(2 \mathrm{H}$, $\left.\mathrm{d}, \mathrm{J}=8.25 \mathrm{~Hz}, \mathrm{H}_{10}\right), 7.79\left(2 \mathrm{H}, \mathrm{d}, \mathrm{J}=8.25 \mathrm{~Hz}, \mathrm{H}_{9}\right)$, 7.90-7.94 (2H, m, $\left.\mathrm{H}_{4,7}\right), 8.03\left(1 \mathrm{H}, \mathrm{d}, \mathrm{J}=2.25 \mathrm{~Hz}, \mathrm{H}_{5}\right)$. ${ }^{13} \mathrm{CNMR}(62 \mathrm{MHz}, \mathrm{DMSO}): \delta_{\mathrm{C}} 16.3\left(\mathrm{CH}_{2}\right), 30.6\left(\mathrm{CH}_{3}\right)$, $32.6(\mathrm{CH} 3), 32.8(\mathrm{CH} 3), 111.5,122.2,124.6,126.8$, 128.7, 132.0, 133.8, 135.5, 140, 140.5, 150.4, 156.1, 159.9 (Alkene and Aryl carbons), $201.7(\mathrm{C}=\mathrm{O})$.

\section{1-(6-((3,4-dimethylphenyl)diazenyl)-2-hydroxy-2-} methyl-2H-chromene-3-yl) ethanone (2e)

IR $(\mathrm{KBr})\left(v_{\max }, \mathrm{cm}^{\prime \prime 1}\right): 3444,1659,1440$, 1165, 1079. ${ }^{1} \mathrm{HNMR}$ (300 MHz, DMSO): $\delta_{\mathrm{H}} 1.86$ $\left(3 \mathrm{H}, \mathrm{s}, \mathrm{CH}_{3}\right), 2.28\left(3 \mathrm{H}, \mathrm{s}, \mathrm{CH}_{3}\right), 2.30\left(3 \mathrm{H}, \mathrm{s}, \mathrm{CH}_{3}\right)$, $2.42\left(3 \mathrm{H}, \mathrm{s}, \mathrm{CH}_{3}\right), 3.4(1 \mathrm{H}, \mathrm{brs}, \mathrm{OH}), 7.08(1 \mathrm{H}, \mathrm{d}, \mathrm{J}$ $\left.=8.7 \mathrm{~Hz}, \mathrm{H}_{8}\right), 7.32\left(1 \mathrm{H}, \mathrm{d}, \mathrm{J}=8.1 \mathrm{~Hz}, \mathrm{H}_{10}\right), 7.58-$ $7.90(4 \mathrm{H}, \mathrm{m}$, Ar Protons), $7.99(1 \mathrm{H}, \mathrm{d}, \mathrm{J}=1.2 \mathrm{~Hz}$, $\left.\mathrm{H}_{5}\right) .{ }^{13} \mathrm{CNMR}$ (75 MHz, DMSO): $\delta_{\mathrm{C}} 22.1\left(\mathrm{CH}_{3}\right), 22.3$ $\left(\mathrm{CH}_{3}\right), 33.5(\mathrm{CH} 3), 33.9(\mathrm{CH} 3), 111,119.5,120.4$, $122.5,125.7,127.3,128.5,132.6,133.7,135$, 139.3, 139.7, 148.9, 156.1, 160.2 (Alkene and Aryl carbons), $201.1(\mathrm{C}=\mathrm{O})$.

1-(6-((4-ethylphenyl)diazenyl)-2-hydroxy-8methoxy-2-methyl-2H-chromene-3-yl) ethanone (2f)

IR $(\mathrm{KBr})\left(v_{\max }, \mathrm{cm}^{-1}\right): 3456,1662,1401$, 1127, 1076. 'HNMR (300 MHz, DMSO): $\delta_{\mathrm{H}} 1.21$ $\left(3 \mathrm{H}, \mathrm{t}, \mathrm{J}=7.5 \mathrm{~Hz}, \mathrm{CH}_{3}\right), 1.89\left(3 \mathrm{H}, \mathrm{s}, \mathrm{CH}_{3}\right), 2.42(3 \mathrm{H}$, s, $\left.\mathrm{CH}_{3}\right), 2.68\left(2 \mathrm{H}, \mathrm{q}, \mathrm{J}=7.8 \mathrm{~Hz}, \mathrm{CH}_{2}\right), 3.3(1 \mathrm{H}, \mathrm{brs}$, $\mathrm{OH}), 3.88\left(3 \mathrm{H}, \mathrm{s}, \mathrm{OCH}_{3}\right), 7.40(2 \mathrm{H}, \mathrm{d}, \mathrm{J}=8.1 \mathrm{~Hz}$, $\left.\mathrm{H}_{10}\right), 7.53\left(1 \mathrm{H}, \mathrm{d}, \mathrm{J}=1.8 \mathrm{~Hz}, \mathrm{H}_{7}\right), 7.69(1 \mathrm{H}, \mathrm{d}, \mathrm{J}=$ $\left.1.8 \mathrm{~Hz}, \mathrm{H}_{5}\right), 7.78\left(2 \mathrm{H}, \mathrm{d}, \mathrm{J}=8.1 \mathrm{~Hz}, \mathrm{H}_{9}\right), 7.87(1 \mathrm{H}$, $\mathrm{s}, \mathrm{H}_{4}$ ).

\section{RESULTS AND DISCUSSION}

Diazotization of substituted aniline and a coupling reaction with 2-hydroxybenzaldehyde or its derivative (3-methoxysalicylaldehyde) yielded<smiles>[R]c1ccccc1/N=N/c1cc([R])c(O)c(C=O)c1</smiles>

Scheme 1: Synthesis of 5-Phenylazo-salicylaldehyde derivatives

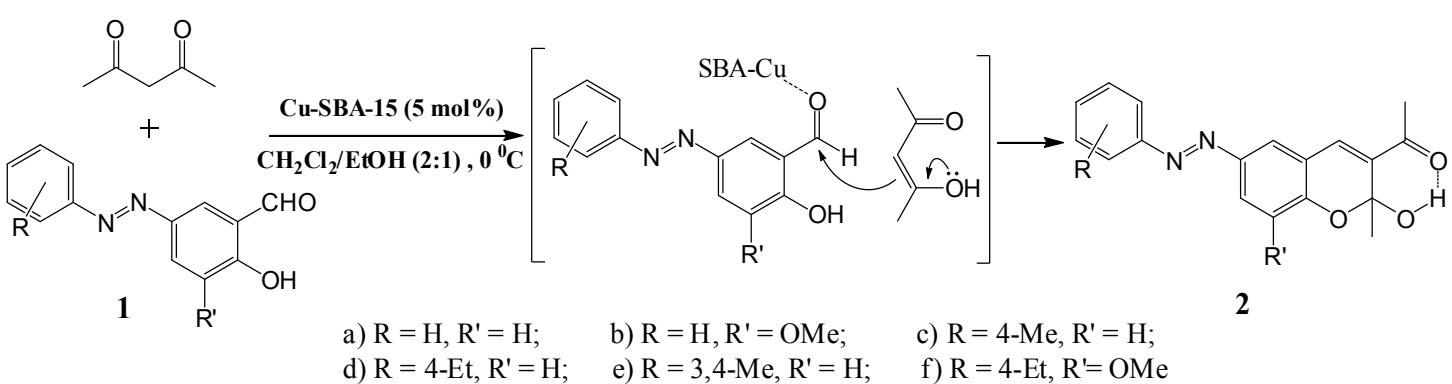

Scheme 2: Synthesis of azo-chromene dyes 
<smiles>[R]c1ccccc1N=Nc1cc([R])c2c(c1)C=C(C(C)=O)C(C)(OO)O2</smiles>

Fig. 1: Number of carbon atoms for structure 2 the corresponding 5-phenylazo-salicylaldehyde (Scheme 1).

Previously, an easy and rapid method for preparation of some $2 \mathrm{H}$-chromene compounds was published ${ }^{36}$ and now we explain a simple and high efficiency pathway for the synthesis of azotated $2 \mathrm{H}$-chromene (2), a novel class of dyes, using mesoporous Cu-SBA-15 as nanocatalyst (Scheme

Table 1 : Yields, mps and Elemental Analysis of 2a-f

\begin{tabular}{lcccccc}
\hline Cmpd & Yields(\%) & mp( ${ }^{\circ} \mathbf{C}$ ) & \multicolumn{3}{c}{ Elemental Analysis (Found) } & Formula (M.W.) \\
& & & $\mathbf{C}$ & $\mathbf{H}$ & $\mathbf{N}$ & \\
\hline $2 \mathrm{a}$ & 88 & $134-136$ & $70.12(70.11)$ & $5.23(5.19)$ & $9.09(9.02)$ & $\mathrm{C}_{18} \mathrm{H}_{16} \mathrm{~N}_{2} \mathrm{O}_{3}(308.33)$ \\
$2 \mathrm{~b}$ & 83 & $141-143$ & $67.44(67.43)$ & $5.36(5.31)$ & $8.28(8.30)$ & $\mathrm{C}_{19} \mathrm{H}_{18} \mathrm{~N}_{2} \mathrm{O}_{4}(338.36)$ \\
$2 \mathrm{c}$ & 85 & $128-130$ & $70.79(70.77)$ & $5.63(5.55)$ & $8.69(8.65)$ & $\mathrm{C}_{19} \mathrm{H}_{18} \mathrm{~N}_{2} \mathrm{O}_{3}(322.36)$ \\
$2 \mathrm{~d}$ & 79 & $121-123$ & $71.41(71.44)$ & $5.99(5.97)$ & $8.33(8.32)$ & $\mathrm{C}_{20} \mathrm{H}_{20} \mathrm{~N}_{2} \mathrm{O}_{3}(336.38)$ \\
$2 \mathrm{e}$ & 81 & $145-147$ & $71.41(71.40)$ & $5.99(5.95)$ & $8.33(8.30)$ & $\mathrm{C}_{20} \mathrm{H}_{20} \mathrm{~N}_{2} \mathrm{O}_{3}(336.38)$ \\
$2 \mathrm{f}$ & 76 & $152-154$ & $68.84(68.81)$ & $6.05(5.99)$ & $7.65(7.59)$ & $\mathrm{C}_{21} \mathrm{H}_{22} \mathrm{~N}_{2} \mathrm{O}_{4}(366.41)$ \\
\hline
\end{tabular}

2). Knoevenagel condensation of compound 1 with acetylacetone yielded compounds $2 \mathrm{a}-2 \mathrm{f}$.

The structures of the new azo chromene dyes were deduced from their IR, ${ }^{1} \mathrm{HNMR},{ }^{13} \mathrm{CNMR}$ spectra, and also $\mathrm{CHN}$ analysis data. The ${ }^{1} \mathrm{H}$ decoupled ${ }^{13} \mathrm{CNMR}$ spectrum for each derivate is completely coincident with the product structure. Due to low solubility, we could not provide ${ }^{13} \mathrm{CNMR}$ spectra for $2 f$ compound.

Fig. 1 illustrates the number of carbon atoms for structure 2. It is helpful for ${ }^{1} \mathrm{HNMR}$ interpretation in spectral data section.

The infrared spectra (IR) of these $2 \mathrm{H}$ chromene dyes show a decreasing intense carbonyl bands due to conjugated carbonyl systems appearing at $1650-1670 \mathrm{~cm}^{-1}$. The broad bands in region of $3250-3480 \mathrm{~cm}^{\prime 1}$ are referred to $v(\mathrm{O}-\mathrm{H})$ vibrations. The peaks appearing at $1000-1200 \mathrm{~cm}^{-1}$ are attributed to $v(\mathrm{C}-\mathrm{O})$ aromatic and aliphatic stretching vibration. The spectra also exhibit the existence of $(-\mathrm{N}=\mathrm{N}-)$ group at $1400-1450 \mathrm{~cm}^{-1}$.

To explain the reaction mechanism, should focus on catalyst. The catalyst has lewis acid sites for polarizing carbonyl groups in both reagents, acetylacetone and 5-phenylazo-salicylaldehyde 1, via the nonbonding electrons on their oxygens. This coordination is more important for acetylacetone because it can lead the equilibrium to enol substance in a suitable solvent.

Since $\beta$-dicarbonyl compounds such as acetylacetone have keto-enol tautomerization, less polar solvents will move the equilibrium toward the enol form. In examine about effect of solvents, better yields were observed by using mixture of dichloromethane and low amount of a protic solvent. 2:1 volume ratio of dichloromethane and ethanol was most suitable according to the high yields (Table 1).

The effect of temperature was also examined. When the reaction warm up to room temperature or higher, the efficiency will decrease. So using ice bath is necessary in whole of the reaction.

\section{CONCLUSIONS}

In summary, a new series of azo-chromene dyes $2 a-f$ were synthesized by use of mesoporous 
nanocatalyst, Cu-SBA-15, leading short reaction times, very simple methodology, high efficiency, easy purification, minimal production of waste and performing reactions in the mild conditions.
However, the diverse characteristics of these azo dye compounds need further investigations.

\section{ACKNOWLEDGMENTS}

We gratefully acknowledge financial supports from the University of Tehran.

\section{REFERENCES}

1. Kang, Y.; Mei, Y.; Du, Y. G.; Jin, Z. Org. Lett. 2003, 5, 4481-4484.

2. Harel, D.; Schepmann, D.; Prinz, H.; Brun, R.; Schmidt, T. J.; Wünsch, B. J. Med. Chem. 2013, 56, 7442-7448.

3. Majumdar, N.; Paul, N. D.; Mandal, S.; Bruin, B.; Wulff, W. D. ACS Catal. 2015, 5, 23292366.

4. Li, B.; Pai, R.; Di, M.; Aiello, D.; Barnes, M. H.; Butler, M. M.; Tashjian, T. F.; Peet, N. P.; Bowlin, T. L.; Moir, D. T. J. Med. Chem. 2012, 55, 10896-10908.

5. Buckingham, R. E.; Clapham, J. C.; Hamilton, T. C.; Longman, S. D.; Norton, J.; Poyaer, R. H. J. Cardiovasc. Pharmacol. 1986, 8, 798804.

6. Lee, B. H.; Seo, H. W.; Yoo, S. E. Pharmacol. 2004, 70, 74-82.

7. Roberston, D. W.; Steinberg, M. I. J. Med. Chem. 1990, 33, 1529-1541.

8. US Patent, 4,716,238, Dec, 29 (1987), Chem. Abstr., $102:$ 45779b (1985).

9. Yue, Y.; Yin, C.; Huo, F.; Chao, J.; Zhang, Y. Sens. Actuator B-Chem. 2016, 223, 496500.

10. Bekhradnia, A.; Domehri, E.; Khosravi, M. Spectrochim. Acta A Mol. Biomol. Spectrosc. 2016, 152, 18-22.

11. Dai, L.; Shi, Y.; Zhao, G.; Shi, M. Chem. Eur. J. 2007, 13, 3701-3706.

12. Yoshioka, E.; Kohtani, S.; Miyabe, H. Angew. Chem. Int. Ed. 2011, 50, 6638-6642.

13. Reddy, K. R.; Poornachandra, Y.; Dev, G. J.; Mallareddy, G.; Nanubolu, J. B.; Kumar, C. G.; Narsaiah, B. Bioorg. Med. Chem. Lett. 2015, 25, 2943-2947.

14. Xia, L.; Cai, H.; Lee, Y. R. Tetrahedron 2015, 71, 6894-6900.

15. Sairam, M.; Saidachary, G.; Raju, B. C.
Tetrahedron Lett. 2015, 56, 1338-1343.

16. Kong, J.; Meng, T.; Su, J. Org. Process Res. Dev. 2015, 19, 681-683.

17. Paul, N. D.; Mandal, S.; Otte, M.; Cui, X.; Zhang, X. P.; Bruin, B. J. Am. Chem. Soc. 2014, 136, 1090-1096.

18. Zonouzi, A.; Izakian, Z.; Biniaz, M. Org. Prep. Proced. Int. 2009, 41, 543-548.

19. Costa, M.; Areias, F.; Abrunhosa, L.; Venancio, A.; Proenca, F. J. Org. Chem. 2008, 73, 19541962.

20. Roy, R.; Rakshit, S.; Bhowmik, T.; Khan, S.; Ghatak, A.; Bhar, S. J. Org. Chem. 2014, 79, 6603-6614.

21. Koukabi, N.; Otokesh, S.; Kolvari, E.; Amoozadeh, A. Dyes Pigm. 2016, 124, 1217.

22. Nisal, A.; Trivedy, K.; Mohammad, H.; Panneri, S.; Gupta, S.; Lele, A.; Manchala, R.; Kumar, N. S.; Gadgil, M.; Khandelwal, H.; More, S.; Laxman, R. S. ACS Sustain. Chem. Eng. 2014, 2, 312-317.

23. Zhao, Y.; Ikeda, T. Smart light-responsive materials: azobenzene-containing polymers and liquid crystals. N.J: Wiley; 2009.

24. Shao, J. Dyes Pigm. 2010, 87, 272-276.

25. Blackburn, O. A.; Coe, B. J. Organometallics 2011, 30, 2212-2222.

26. Cheng, X.; Li, Q.; Li, C.; Qin, J.; Li, Z. Chem. Eur. J. 2011, 17, 7276-7281.

27. Li, T.; Yang, Z.; Li, Y.; Liu, Z.; Qi, G.; Wang, B. Dyes Pigm. 2011, 88, 103-108.

28. Bogdanov, A. V.; Vorobiev, A. K. J. Phys. Chem. B 2013, 117, 13936-13945.

29. Banerjee, I. A.; Yu, L.; Matsui, H. J. Am. Chem. Soc. 2003, 125, 9542-9543.

30. Kundu, S. K.; Mondal, J.; Bhaumik, A. Dalton Trans. 2013, 42, 10515-10524.

31. Dutta, A.; Mondal, J.; Patra, A. K.; Bhaumik, 
A. Chem. Eur. J. 2012, 18, $13372-13378$.

32. Mondal, J.; Modak, A.; Dutta, A.; Bhaumik, A. Dalton Trans. 2011, 40, 5228-5235.

33. Li, J.; Li, X.; Wang, S. J. Mol. Struct. 2012, 1011, 19-24.

34. Manimekalai, A.; Balachander, R. J. Mol. Struct. 2012, 1027, 175-185.
35. Chen, L.; Guo, P.; Zhu, L.; Qiao, M.; Shen, W.; Xu, H.; Fan, K. Appl. Catal. A. Gen. 2009, 356, 129-136.

36. Zonouzi, A.; Googheri, M. S.; Miralinaghi, P. S. Org. Prep. Proced. Int. 2008, 40, 471-477. 